\section{Extrema resistência à insulina subcutânea e intramuscular em diabetes melito tipo 1}

\author{
Extreme subcutaneous and intramuscular insulin \\ resistence at type 1 diabetes mellitus
}

Anielli Pinheiro', Lúcia H. B. Tácito', Antônio C. Pires

\section{SUMÁRIO}

Resistência insulínica consiste em reduzida resposta celular a esse hormônio e, portanto, disfunção do transporte de glicose para o meio intracelular. Esse fenômeno associa-se a fatores genéticos e principalmente comportamentais relacionados a obesidade e comorbidades a ela associadas como diabetes melito tipo 2, hipertensão arterial e dislipidemia. Entretanto, fatores clínicos de resistência insulínica também estão presentes em diabéticos tipo 1 não obesos na conhecida síndrome de extrema resistência à insulina subcutânea e intramuscular (DRIASM). Condição rara que consiste em resistência à ação da insulina no tecido subcutâneo e muscular e sensibilidade normal, ou próxima do normal, quando administrada via intravenosa. Tratamentos propostos até o momento mostram-se pouco efetivos e se relacionam a complicações e faIhas frequentes. Descrevemos dois casos de pacientes femininas de 45 e 46 anos com DRIASM que se diferenciam dos demais já descritos por apresentar diagnóstico de diabetes melito tipo 1 tardio, hiperglicemia constante associada a complicações catabólicas, microvasculares (retinopatia) e neuropáticas sem, no entanto, nenhum episódio de cetoacidose diabética. Os tratamentos propostos variaram desde aplicação de insulina intramuscular e intravenosa até listagem para possível realização de transplante de pâncreas como tentativa de tratamento definitivo. Este trabalho teve aprovação do Comitê de Ética em Pesquisa da Faculdade de Medicina de São José do Rio Preto. Arq Bras Endocrinol Metab. 2011;55(3):233-7

\section{SUMMARY}

Insulin resistance signs reduced cellular response to this hormone and dysfunction of glucose transport to intracellular compartment. This phenomenon is associated to genetic factors and principally behavior factors correlating to obesity and its comorbidities, as type 2 diabetes mellitus, hypertension and dyslipidemia. However clinical factors of insulin resistance are still present at not obese type 1 diabetes in a known syndrome called type 1 diabetes mellitus with resistance to insulin administered subcutaneously and intramuscularly (DRIASM). This is a rare condition that consists into insulin resistance at subcutaneously and intramuscularly use and normal or near to normal sensitivity at intravenously way. Treatments until now proposed are ineffective and are related to frequent fails and complications. We report here two cases of DRIASM in 45 and 46 female patients that are different from others yet related because they have late diabetes type 1, sustained hyperglycemia associated to catabolic, microangiopathy and neuropathic complications without any ketoacidosis episode. The treatment vary from alternative ways for insulin infusion to inscription to a possible performance of pancreas transplantation like a experiment of definitive treatment. This report was approved by Research Ethic Committee from São José do Rio Preto medical school. Arq Bras Endocrinol Metab. 2011;55(3):233-7
${ }^{1}$ Disciplina de Endocrinologia e Metabologia, Faculdade de Medicina de São José do Rio Preto (Famerp), São José do Rio Preto, SP, Brasil

\section{INTRODUÇÃO}

$\mathrm{A}$ resistência insulínica consiste em reduzida resposta celular a este hormônio e consequente disfunção no transporte de glicose para o meio intracelular. Associa-se a fatores genéticos e principalmente comportamentais relacionados a obesidade e comorbidades como diabetes melito tipo 2 (DM 2), hipertensão arterial sistêmica e dislipidemia. Entretanto, os sinais clínicos de resistência 
insulínica podem ser encontrados em indivíduos não obesos e portadores de diabetes melito tipo l (DM l), caracterizando a chamada síndrome de extrema resistência à insulina (1). Essa rara condição manifesta-se com resistência à ação da insulina no tecido subcutâneo (SC), com sensibilidade normal, ou próxima do normal, quando administrada de forma contínua por via endovenosa (2). Além disso, a resistência insulínica pode apresentar-se concomitantemente por via intramuscular (IM) caracterizando o DRIASM (3).

$\mathrm{Na}$ tentativa de explicar o DRIASM, teorias recentes discutem a possibilidade de número reduzido de receptores ou disfunção dos mecanismos pós-receptores de insulina como possíveis fatores etiológicos (1). Outra possibilidade seria o aumento da degradação da insulina no tecido subcutâneo (2) pela ação de duas principais enzimas: insulinase e proteína dissulfide isomerase (3). No entanto, sua fisiopatologia permanece desconhecida e por isso inúmeros tratamentos propostos até o momento mostram-se ineficazes (4). Diante do exposto, descrevemos dois casos de DRIASM nos quais os tratamentos propostos variaram desde aplicação de insulina IM e endovenosa (EV) até listagem para possível transplante isolado de pâncreas como tentativa de tratamento definitivo.

\section{CASO 1}

R.M.S.P., 45 anos, feminina, negra, procedente de São José do Rio Preto, encaminhada ao ambulatório de Endocrinologia de nossa Instituição em 2007, pois teve diagnóstico de diabetes melito em 2001 e apresentava difícil controle há 8 meses. Na primeira consulta, apresentava complicações como retinopatia diabética e quadro diarreico crônico, além de hiperglicemias frequentemente associadas a episódios de síncope com vários atendimentos em setor de emergências de Unidades Básicas de Saúde da região. A história clínica compreendia sinais e sintomas como perda ponderal de $30 \mathrm{~kg}$ no período, polidipsia e polifagia. $\mathrm{Na}$ ocasião, com $55 \mathrm{~kg}$ e índice de massa corpórea (IMC) de $22 \mathrm{~kg} / \mathrm{m}^{2}$, encontrava-se em uso de doses diárias de $150 \mathrm{UI}$ de insulina $\mathrm{NPH}$ e 50 UI de insulina regular SC, além de 2.550 mg de metformina, mantendo valores glicêmicos à monitorização domiciliar sempre maiores que $450 \mathrm{mg} / \mathrm{dL}$, com valores muitas vezes acima de $600 \mathrm{mg} / \mathrm{dL}$ (HI). Durante o seguimento ambulatorial, foram prescritos reajustes progressivos de insulina, chegando a usar doses diárias de 400 UI de insulina NPH e 150 UI de in- sulina regular SC, associadas a $100 \mathrm{mg}$ de vildagliptina. A dosagem de anticorpos antidescarboxilase de ácido glutâmico (anti-GAD) foi negativa $(0,2)$ e de peptídeo C, baixa $(1,5 \mathrm{ng})$, tornando possível diagnóstico de DM 1. O uso de glicocorticoides e a tentativa de administração de insulina via intramuscular mostraram-se sem sucesso no controle glicêmico. No ano de 2008, necessitou de intervenção cirúrgica ginecológica, sendo submetida à insulinoterapia utilizando bomba de infusão endovenosa contínua, atingindo pela primeira vez controle glicêmico adequado, embora com doses médias maiores que as habituais $(0,3 \mathrm{UI} / \mathrm{kg} / \mathrm{h}$ de insulina regular). Recebeu alta após sete dias do procedimento utilizando insulina regular endovenosa com aplicações intermitentes em cateter venoso central (Portocath), meio que utilizou por seis meses até apresentar infecção de corrente sanguínea obrigando sua retirada. Atualmente, segue estável do ponto de vista clínico, apresentando adequado controle glicêmico em uso de insulinoterapia $\mathrm{EV}$, administrada pela própria paciente por meio de acesso venoso periférico. Evoluiu com ganho ponderal de $3 \mathrm{~kg}$ em 6 meses e não apresentou novos episódios de diarreia ou perda de consciência.

\section{CASO 2}

A.P.P.N., 45 anos, feminina, negra, portadora de DM 1 desde 2006 e em acompanhamento ambulatorial regular com nossa equipe desde outubro de 2008 em decorrência de intenso descontrole glicêmico há aproximadamente seis meses. Inicialmente apresentou quadro de poliúria, polidipsia, polifagia e perda ponderal de $36 \mathrm{~kg}$ associado a valores glicêmicos em torno de $400 \mathrm{mg} / \mathrm{dL}$ em diversos horários. Nos primeiros dois anos de seguimento, na cidade de origem (Penápolis - SP), a paciente utilizou doses diárias de 145 UI de insulina NPH SC, associada a $850 \mathrm{mg}$ de metformina, porém, como ainda mantinha controle glicêmico inadequado, optou-se por encaminhá-la ao ambulatório de Endocrinologia do Hospital de Base da Faculdade de Medicina de São José do Rio Preto. Na primeira consulta, apresentava-se com $52 \mathrm{~kg}$ e IMC de $19,6 \mathrm{~kg} / \mathrm{m}^{2}$, glicemia de jejum de 366 $\mathrm{mg} / \mathrm{dL}, \mathrm{HbAlc}$ de $16,8 \%$ e retinopatia diabética não proliferativa. Optou-se por manter a dose usual de insulina NPH associada a $1.700 \mathrm{mg} /$ dia de metformina. A positividade para anticorpo antiGAD $(55,2 \mathrm{U} / \mathrm{mL}) \mathrm{e}$ peptídeo $\mathrm{C}(0, \mathrm{lng} / \mathrm{mL})$ baixo confirmou diagnóstico de DM 1. Em fevereiro de 2009, foi internada para tratamento com infusão de insulina regular EV em bom- 
ba de infusão contínua, considerando a glicotoxicidade como fator preponderante ao seu inadequado controle glicêmico. Em 24 horas, recebeu 214 UI de insulina regular endovenosa, com valores glicêmicos variando entre 39 e $246 \mathrm{mg} / \mathrm{dL}$. Recebeu alta hospitalar com prescrição de doses diárias SC de 100 UI de glargina SC e 30 UI de insulina regular. Como não manteve adequado o controle glicêmico com o uso de insulina SC, foi proposta nova internação na tentativa de administração de insulina NPH IM considerando a hipótese de resistência subcutânea à ação da insulina, e os resultados anteriores bem-sucedidos com infusão via endovenosa. Na admissão, apresentava glicemia de $420 \mathrm{mg} / \mathrm{dL}$ e, durante as primeiras 24 horas de internação, recebeu um total de 60 UI de insulina NPH via IM, apresentando então média glicêmica de $86,1 \mathrm{mg} / \mathrm{dL}$, com variação entre 43 e $180 \mathrm{mg} / \mathrm{dL}$. Recebeu alta hospitalar com dose diária de 20 UI IM de glargina, seguindo acompanhamento ambulatorial com razoável controle glicêmico até o início de maio de 2009, quando retornou ao serviço queixando-se de quadro de diarreia líquida, explosiva, com episódios predominando no período noturno. Recebeu tratamento para verminoses, sendo realizada investigação clínica considerando-se os diagnósticos diferenciais de doença celíaca e gastroparesia com proliferação bacteriana. Em julho do mesmo ano, retornou ao ambulatório referindo dois episódios de síncope associados a hiperglicemias capilares, com valores acima de $600 \mathrm{mg} / \mathrm{dL}$, (HI) em todos os horários monitorizados, além de perda ponderal involuntária de $5 \mathrm{~kg}$ e piora dos episódios de diarreia. Diante desse quadro de descontrole metabólico e catabolismo e considerando-se o insucesso da terapêutica proposta, foi iniciada insulinoterapia EV com múltiplas aplicações diárias (a cada quatro horas) por acesso venoso periférico inicialmente, seguido de implante de acesso venoso central. A paciente apresentou melhora dos parâmetros glicêmicos e da sintomatologia referida. Também apresentou infecção de corrente sanguínea associada a cateter central e voltou a utilizar via endovenosa periférica.

Os valores glicêmicos relacionados às doses de insulina e via de administração utilizada estão representados nas figuras 1 e 2, respectivamente, para os casos homônimos.

\section{DISCUSSÃO}

Este estudo descreve duas pacientes com extrema resistência à insulina (DRIASM) que se assemelham quanto ao grupo étnico, baixos valores de peptídeo C, hiperglicemias frequentes refratárias aos procedimentos habituais e ausência de condições clássicas relacionadas à resistência insulínica como obesidade, dislipidemia e hipertensão arterial sistêmica. DRIASM é condição extremamente rara com menos de 30 casos descritos em literatura (3) e foi definida por Paulsen e cols. (5) em 1979 como resistência à ação hipoglicemiante da insulina por via subcutânea, mas não EV, associado à ausência de aumento da insulinemia após injeção de insulina SC decorrente do possível aumento da atividade enzimática que degrada a insulina no SC. Diferentemente de outros casos descritos, as duas pacientes acima tiveram diagnóstico de DM 1 tardio, sendo que para uma delas o DRIASM foi a manifestação inicial da doença e, além disso, não apresentaram nenhum episódio de cetoacidose diabética ou estado hiperglicêmico hiperosmolar como complicações (3). Demonstraram alto grau de catabolismo proteico, ilustrando complicações autonômicas provavelmente decorrentes do extremo descontrole glicêmico em longo prazo. No contexto, os "clampes" euglicêmicos e hiperinsulinêmicos comprovaram a sensibilidade quase normal à ação da insulina por via endovenosa para ambas as pacientes.

As possibilidades terapêuticas propostas nessas condições consistem em vias alternativas para administração de insulina, sendo a via intramuscular considerada por Pickup e cols. (6), Dandona e cols. (7), Brossard e cols. (8) e Larsen (9), a via endovenosa ou intraperitoneal por Schade e cols. (10), Campbell e cols. (11), Dandona e cols. (7) e Riveline e cols. (4,12). Alguns pacientes foram submetidos ao tratamento com inibidores de protease com respostas satisfatórias (13-15). Entretanto, constituem soluções apenas temporárias, levando-se em consideração sua associação a recorrentes infecções de corrente sanguínea e respostas progressivamente reduzidas do controle glicêmico (4). Nesse contexto de difícil controle do diabetes, o transplante isolado de pâncreas surge como potencial alternativa terapêutica. Apesar da falta de evidências claras, essa indicação a pacientes com extrema resistência à insulina e estado hiperglicêmico refratário a tratamentos convencionais pode trazer benefícios clínicos que suplantam as complicações relacionadas à imunossupressão prolongada e demais riscos do procedimento cirúrgico (9). O acompanhamento desses casos em longo prazo associado a novos estudos torna-se necessário para predizer a eficácia dessa nova proposta de indicação dessa modalidade terapêutica para a síndrome de extrema resistência insulínica. 
Paciente 1

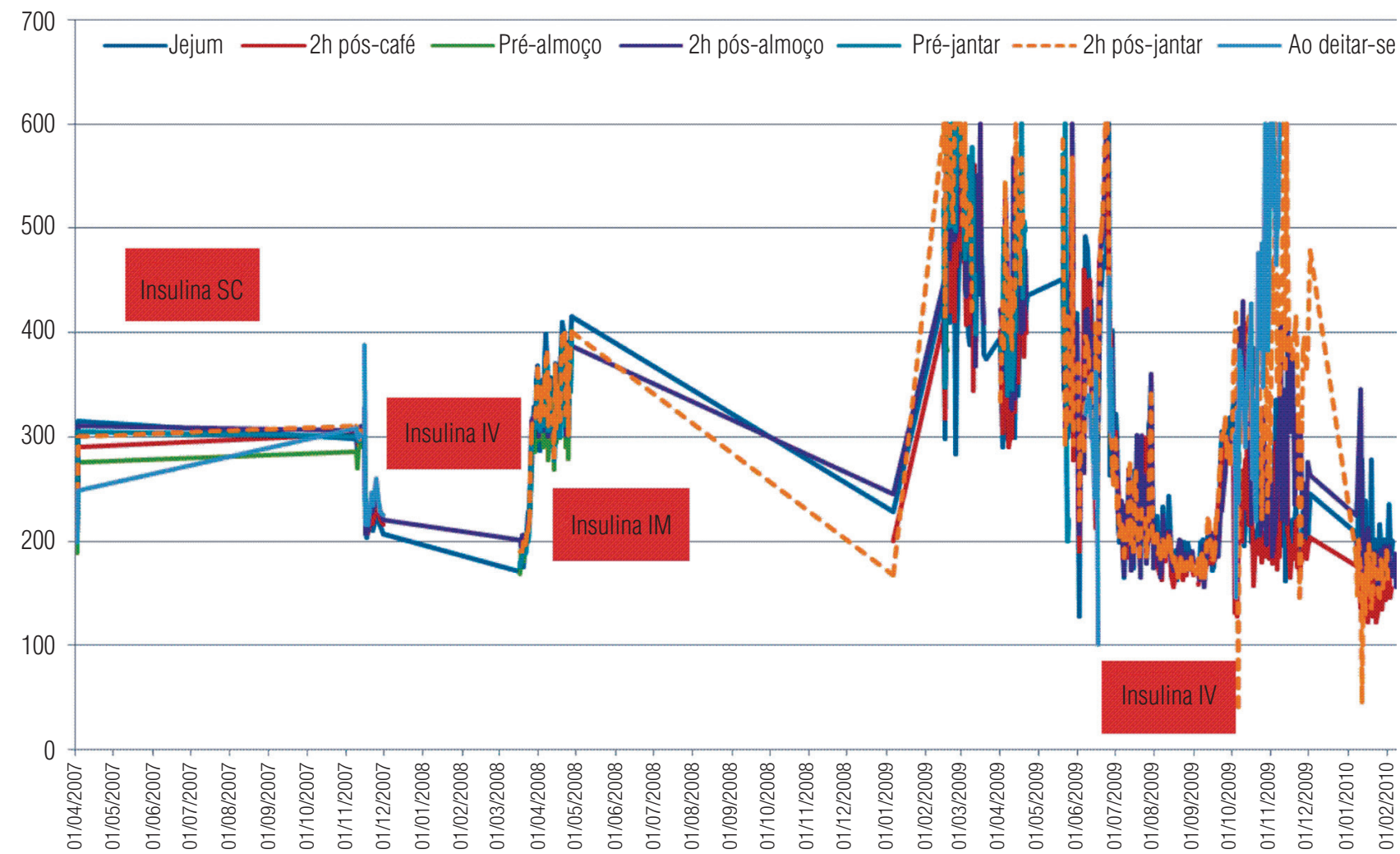

Figura 1. Linhas do tempo relacionando perfil glicêmico X vias de aplicação de insulina.

Paciente 2

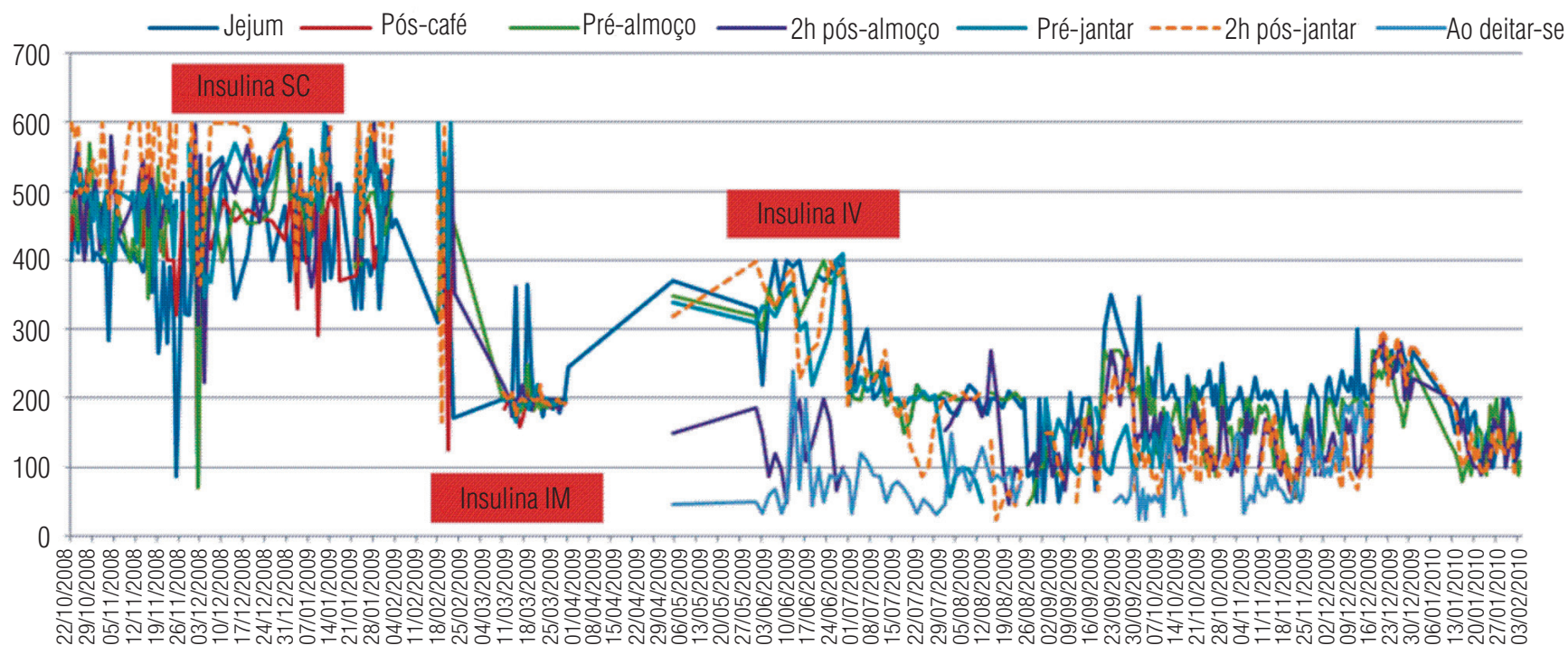

Figura 2. Linhas do tempo relacionando perfil glicêmico X vias de aplicação de insulina. 
Agradecimentos: Os autores gostariam de agradecer ao estatístico Prof. Dr. José Antonio Cordeiro por sua assistência na análise dos dados.

Declaração: os autores declaram não haver conflitos de interesse científico neste estudo.

\section{REFERÊNCIAS}

1. Uruska A, Araszkiewicz A. Insulin resistance in patients with type 1 diabetes. Pediatr Endocrinol Diabetes Metab. 2009;15(2):119-23.

2. Soudan B, Girardot C, Fermon C. Extreme subcutaneous insulin resistance: a misunderstood syndrome. Diabetes Metab. 2003;29:539-46.

3. Sa JR, Alvarenga MA, Rangel EB, Melaragno CS, Gonzalez AM, Linhares MM, et al. Extreme subcutaneous, intramuscular and inhaled resistance treated by pancreas transplantation alone. Am JTransplant. 2010;10:184-8.

4. Riveline JP, Vantyghem MC, Fermon C, Brunet C, Gautier JF, Renard E, et al; EVADIAC group. Subcutaneous insulin resistance successfully circumvented on long term by peritoneal insulin delivery from a implantable pump in four diabetic patients. Diabetes Metab. 2005:31:496-8.

5. Paulsen EP, Courtney JW III, Duckworth WC. Insulin resistance caused by a massive degradation of subcutaneous insulin. Diabetes. 1979;28:640-5.

6. Pickup JC, Home PD, Bilous RW, Keen H, Alberti KG. Management of severely brittle diabetes by continuous subcutaneous and intramuscular insulin infusions: evidence for a defect in subcutaneous insulin absorption. Br Med J (Clin Res Ed). 1981;282:347-50.
7. Dandona P, Fonseca V, Fernando O, Menon RK, Weerakoon J, Kurtz A, et al. Control of diabetes through a subcutaneous peritoneal access device (SPAD) in patients with resistance to subcutaneously injected insulin. Diabetes Res. 1987;5:47-9.

8. Brossard JH, Havrankova J, Rioux D, Bertrand S, D'Amour P. Longterm use of intramuscular insulin therapy in a type I diabetic patient with subcutaneous insulin resistance. Diabet Med. 1993;10:174-6

9. Larsen JN. Transplantation: indications and consequences. Endocr Rev. 2004;25(6):919-46.

10. Schade DS, Eaton RP, Warhol RM, Gregory JA, Doberneck RC. Subcutaneous peritoneal access device for type I diabetic patients nonresponsive to subcutaneous insulin. Diabetes. 1982;31:470-3.

11. Campbell IW, Kritz H, Najemnik C, Hagmueller G, Irsigler K. Treatment of a type I diabetic with subcutaneous insulin resistance by a totally implantable insulin infusion device ("Infusaid"). Diabetes Res. 1984;1:83-8.

12. Riveline JP, Capeau J, Robert JJ, Varroud-Vial M, Cerf-Baron I, Deburge $A$, et al. Extreme subcutaneous insulin resistance successfully treated by an implantable pump. Diabetes Care. 2001;24:2155-6.

13. Muller WA, Taillens $C$, Léreret $S$, Berger M, Philippe J, Halban PA, et al. Resistance against subcutaneous insulin successfully managed by aprotinin. Lancet. 1980;7:1245-6.

14. Freidenberg GR, White N, Cataland S, O'Dorisio TM, Sotos JF, Santiago JV. Diabetes responsive to intravenous but not subcutaneous insulin: effectiveness of aprotinin. N Engl J Med. 1981;305:363-8.

15. Maberly GF, Wait GA, Kilpatrick JA, Loten EG, Gain KR, Stewart, et al. Evidence for insulin degradation by muscle and fat tissue in an insulin resistant diabetic patient. Diabetologia. 1982;23:333-6. 